\title{
Review
}

\section{Another way to die: autophagic programmed cell death}

\author{
Y Tsujimoto*,1,2 and S Shimizu ${ }^{1,2}$ \\ 1 Laboratory of Molecular Genetics, Department of Medical Genetics, Osaka \\ University Medical School, 2-2 Yamadaoka, Suita, Osaka 565-0871, Japan \\ 2 SORST of Japan Science Technology Corp., 2-2 Yamadaoka, Suita, Osaka \\ 565-0871, Japan \\ * Corresponding author: Y Tsujimoto, Laboratory of Molecular Genetics, \\ Department of Medical Genetics, Osaka University Medical School, 2-2 \\ Yamadaoka, Suita, Osaka 565-0871 Japan. Tel: + 81-6-6879-3360; \\ Fax: +81-6-6879-3369; E-mail: tsujimot@gene.med.osaka-u.ac.jp
}

Received 06.5.05; revised 18.8.05; accepted 18.8.05

Edited by G Melino

\begin{abstract}
Programmed cell death (PCD) is one of the important terminal paths for the cells of metazoans, and is involved in a variety of biological events that include morphogenesis, maintenance of tissue homeostasis, and elimination of harmful cells. Dysfunction of PCD leads to various diseases in humans, including cancer and several degenerative diseases. Apoptosis is not the only form of PCD. Recent studies have provided evidence that there is another mechanism of PCD, which is associated with the appearance of autophagosomes and depends on autophagy proteins. This form of cell death most likely corresponds to a process that has been morphologically defined as autophagic PCD. The present review summarizes recent experimental evidence about autophagic PCD and discusses some aspects of this form of cell death, including the mechanisms that may distinguish autophagic death from the process of autophagy involved in cell survival.

Cell Death and Differentiation (2005) 12, 1528-1534.

doi:10.1038/sj.cdd.4401777
\end{abstract}

Keywords: cell death; autophagy; apoptosis; Bcl-2; Bax

Abbreviations: PCD, programmed cell death; MEFs, mouse embryonic fibroblasts; 3-MA, 3-methyladenine; WT, wild-type; Glud2, glutamate receptor $\delta 2$

\section{A Constantly Raised Concern for Autophagic Death}

Apoptosis, a form of programmed cell death (PCD), has attracted considerable attention over the last 15 years and a significant part of the molecular mechanisms involved has already been unveiled. However, there is considerable evidence to suggest that PCD is not confined to apoptosis and that other mechanisms may also operate. One of these mechanisms is the so-called 'autophagic PCD', ${ }^{1}$ which is a process that is associated with autophagosomes and autolysosomes.

\section{Autophagy}

Here, we briefly summarize the well-established process of autophagosome formation. Readers who are interested in more detailed information on autophagy should refer to several excellent recent reviews. ${ }^{2-4}$

Autophagosomes are double-membrane cytoplasmic vesicles that are designed to engulf various cellular constituents, including cytoplasmic organelles (Figure 1). Autophagosomes fuse to lysosomes to become autolysosomes, where sequestered cellular components are digested. Autophagosomes and autolysosomes are formed during a process called macroautophagy (hereafter this is referred to as 'autophagy'), which is activated by starvation conditions associated with deficiency of nutrients such as amino acids. Autophagy is also known to be activated by hypoxic conditions and high temperatures. Autophagy is a process by which cells undergo partial autodigestion (the term is derived from ancient Greek meaning to 'eat oneself') that prolongs survival for a short time under starvation conditions. It provides nutrients that are necessary to maintain cell viability. It has more recently been shown that autophagy is also involved in the killing of bacteria that are ingested by cells. ${ }^{5,6}$

The molecular basis of autophagy has been extensively studied, mainly in yeasts, by investigation of autophagydefective mutants to identify the responsible genes (designated as atg). It is currently known that the basic mechanism of autophagy has been well conserved during evolution from the fact that diverse organisms, including yeasts, flies, and mammals, all carry a similar set of atg genes, although there are some significant differences between yeasts and higher eukaryotes. Autophagy is regulated by PI3 kinase type I and III. PI3 kinase type I is activated by growth factors like insulin and inhibits autophagy through PDK1 and AKT, which regulates mammalian target of rapamycin (mTOR). It is still unclear how activation of mTOR inhibits autophagy, but the mechanism may involve phosphorylation of Atg13, which is part of a protein complex with Atg1, a serine/threonine kinase. Since mTOR is regulated by many other proteins, regulation of the process of autophagy is likely to be very complex. PI3 kinase type III, which includes Atg6 in its complex, promotes the nucleation of autophagic vesicles (Figure 1). Expansion of autophagic vesicles is mediated by two ubiquitin-like conjugation systems: (1) the Atg12 pathway (involving Atg12 (ubiquitin-like), Atg7 (E1-like), Atg10 (E2-like), and Atg5) and (2) the Atg8 pathway (involving Atg8 (ubiquitin-like), Atg7 (E1-like), Atg3 (E2-like), and Atg4) (Figure 1). In the Atg12 pathway, Atg12 is conjugated to Atg5, while Atg8 is conjugated to phosphatidylethanolamine in the Atg8 pathway. 


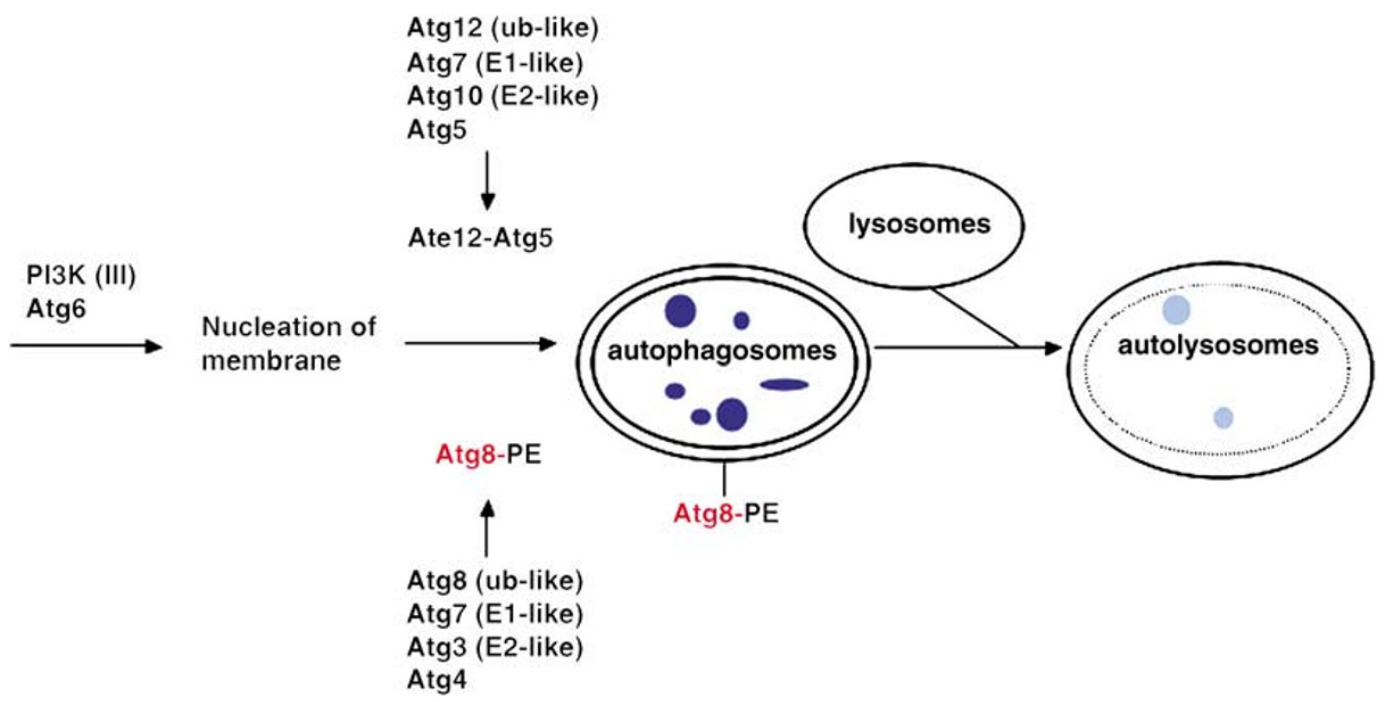

Figure 1 Diagram of the autophagic process. PI3 kinase type III and two ubiquitin-like conjugation systems are involved in the formation of autophagosomes. PE: phosphatidylethanolamine; ub: ubiquitin

\section{Autophagic death}

Autophagic cell death is mainly a morphologic definition (i.e. cell death associated with autophagosomes/autolysosomes), and there is still no conclusive evidence that a specific mechanism of autophagic death actually exists. However, it is quite conceivable that constitutive autophagy could eventually destroy a cell. This hypothesis as well as previous reports that cells with autophagic features often exist in regions where PCD is occurring seem to support the existence of autophagic cell death. ${ }^{7}$ One question that constantly arises, however, is whether autophagic activity in dying cells is the cause of death or is actually an attempt to prevent it. Morphological and histochemical studies cannot prove a causative relationship between the autophagic process and cell death. In fact, there have recently been strong arguments that autophagic activity in dying cells might actually be a survival mechanism.

One reason why this issue has not been resolved is a lack of a suitable experimental system to investigate autophagic death. We recently found that cytotoxic stimuli activate autophagic death in cells that are protected against apoptosis, such as those expressing antiapoptotic $\mathrm{Bcl}-2$ or $\mathrm{Bcl}-\mathrm{x}_{\mathrm{L}}$, or those lacking both Bax and Bak (multidomain proapoptotic members of the Bcl-2 family that function as a gateway for a variety of apoptotic signals). ${ }^{8}$ Lenardo's group has also found that cell lines such as L929 undergo autophagic death in the presence of z-VAD-fmk, a pancaspase inhibitor. ${ }^{9}$

\section{Autophagic Programmed Death is an Alternative to Apoptosis}

Bax/Bak-deficient mouse embryonic fibroblasts (MEFs) do not undergo apoptosis after exposure to a variety of apoptotic stimuli. ${ }^{10,11}$ Instead, we have found that these cells die in a nonapoptotic manner when exposed to various cytotoxic agents, such as etoposide, staurosporine, and thapsigargin. MEFs exposed to these agents show (1) loss of clonogenicity (the ability to form colonies) and (2) positive staining with
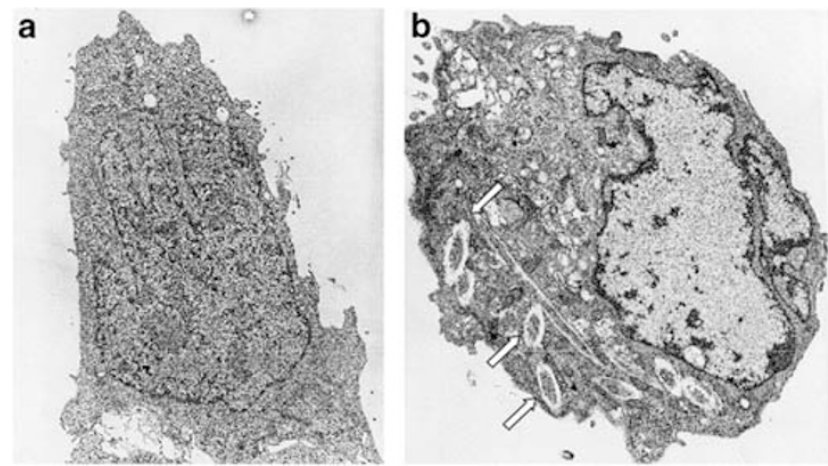

Figure 2 Electron micrograph of Bax/Bak-deficient MEFs with and without exposure to etoposide. (a) Control cell and (b) etoposide $(20 \mu \mathrm{M})$-treated cell. The etoposide-treated cell contains many autophagosomes (arrows)

propidium iodide (a marker of plasma membrane disruption), findings which are compelling evidence of cell death when taken together. ${ }^{8}$ Electron microscopy has revealed that drugtreated Bax/Bak-deficient cells contain numerous doublemembrane vesicles ${ }^{8}$ (also see Figure 2), which have been confirmed to be autophagosomes by the punctate distribution of GFP-LC3 (GFP-Atg8: a portion specifically concentrated on autophagosomes that is normally spread throughout the cytoplasm) ${ }^{8}$ (also see Figure 3). This nonapoptotic form of cell death can be inhibited by suppressing autophagosome formation with autophagy inhibitors, such as 3-methyladenine (3-MA) and wortmannin, or by silencing Atg5 and Atg6, as assessed by several different methods. ${ }^{8}$ Inhibition of cell death has been convincingly demonstrated by detection of improved clonogenicity ${ }^{8}$ (also see Figure 4). Thus, these results indicate that autophagosome formation is required for cells to die after exposure to cytotoxic drugs, proving the existence of an alternative death mechanism to apoptosis that could be termed autophagic cell death. Independently, Lenardo's group has also shown by studies on several cell lines, including mouse L929 cells and human U937 cells, that 

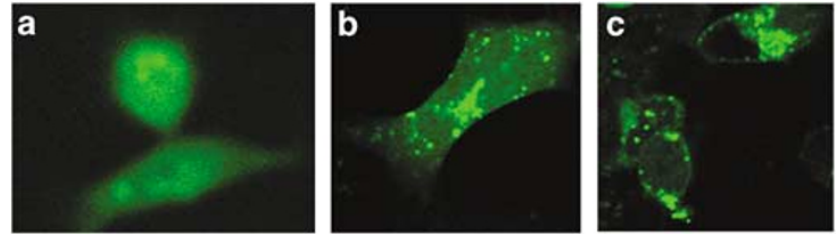

Figure 3 GFP-LC3 fluorescence in Bax/Bak-deficient MEFs. Bax/Bak-deficient MEFs were transfected with GFP-LC3 DNA and then cultured (a) without etoposide, (b) with etoposide $(20 \mu \mathrm{M})$, and (c) in the absence of amino acids. Whereas untreated healthy cells show diffuse distribution of GFP-LC3, Bax/Bakdeficient MEFs exposed to etoposide or amino-acid depletion show a punctate distribution of GFP-LC3, indicating the formation of autophagosomes. LC3 is known to be concentrated on autophagosomes

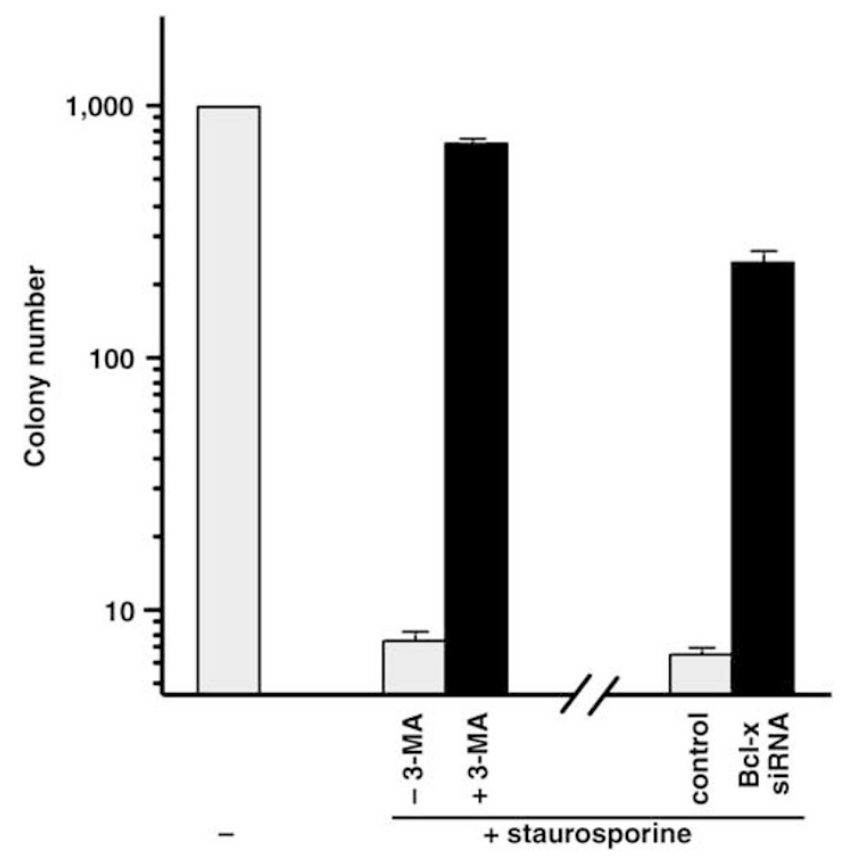

Figure 4 Inhibition of autophagic death in Bax/Bak-deficient MEFs by an autophagy inhibitor and by silencing of Bcl-x. Bax/Bak-deficient MEFs were left untreated $(-)$ or treated $(+)$ with $1 \mu \mathrm{M}$ staurosporine for $24 \mathrm{~h}$ in the absence or presence of $10 \mathrm{mM}$ 3-methyladenine (3-MA). Cells were also treated with and without $\mathrm{Bcl}-\mathrm{x}$ siRNA and then with $1 \mu \mathrm{M}$ staurosporine for $24 \mathrm{~h}$. Cell viability was assessed by a clonogenicity assay: cells were washed and replated in regular medium to allow the formation of colonies

a pancaspase inhibitor induces nonapoptotic death associated with autophagic manifestations, which is inhibited by silencing of Atg5 and Atg7. ${ }^{9}$

Based on our observations and Lenardo's, we propose that a better definition of autophagic cell death would include an association with autophagosomes as well as dependence on autophagy proteins. In this regard, the death of Purkinje cells induced by the Lurcher mutation (a constitutive active mutation) of the $\delta 2$ glutamate receptor (Glud2) could be categorized as a type of autophagic death because it is associated with autophagosomes and is inhibited by 3-MA. ${ }^{12}$ It has been shown that Glud2 interacts with nPIST (a PDZ protein specifically interacting with $\mathrm{TC} 10$ ) that binds to Beclin 1 (Atg6), suggesting a molecular link between Glud2 and the a
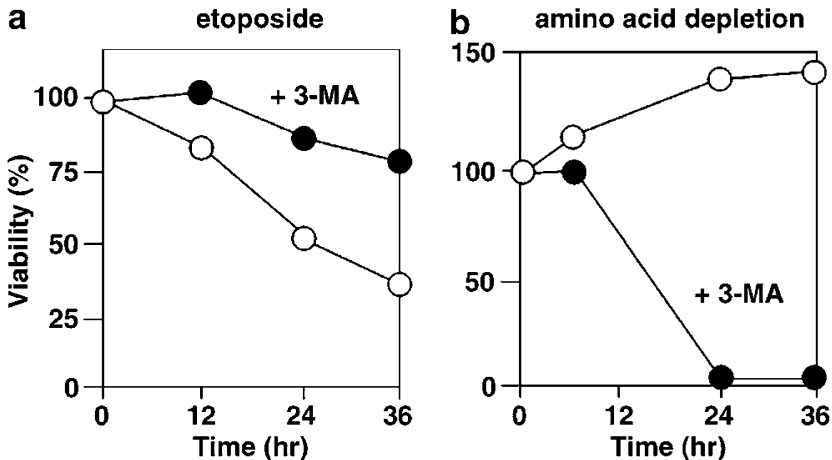

Figure 5 Inhibition of etoposide-induced death, but not amino-acid depletioninduced death, of Bax/Bak-deficient MEFs by 3-MA. Bax/Bak-deficient MEFs were incubated with $20 \mu \mathrm{M}$ etoposide (a) or in the absence of amino acids (b) in the presence and absence of $10 \mathrm{mM}$ 3-MA for the indicated times, and cell viability was assessed by the Cell Titer Blue assay ${ }^{8}$

autophagic machinery. ${ }^{12}$ It was also recently reported that INF $\gamma$ induces Atg5-dependent autophagic death. ${ }^{13}$ On the other hand, the following situation should not be considered as autophagic death: when Bax/Bak-deficient MEFs are cultured in the absence of amino acids, the process of autophagy is activated to prolong survival (Figure 3c), but the cells eventually die of nutrient deficiency unless amino acids are provided. In this setting, inhibition of autophagy actually enhances cell death, indicating that such death does not depend on autophagy proteins (Figure 5). The same is true for the death of an IL3-dependent cell line induced by IL-3 deprivation, because autophagy is activated but does not itself cause death. ${ }^{14}$ In these situations, autophagy is activated as a mechanism that prolong cell survival (Figure 6). We will discuss this issue further in the following sections.

Although it has been shown that Bax/Bak-deficient MEFs undergo autophagic death after exposure to etoposide, staurosporine, and thapsigargin, ${ }^{8}$ neither $\mathrm{X}$-ray irradiation nor TNF/cycloheximide induces autophagic death (our unpublished results), even though all of these stimuli induce apoptosis of wild-type (WT) MEFs. Similarly, in Bax/Bakdeficient thymocytes, autophagic death is readily induced by etoposide and staurosporine, ${ }^{8}$ but not by dexamethasone (our unpublished results), although all of these reagents induce apoptosis in WT thymocytes. These results indicate that only certain death stimuli trigger the autophagic death of Bax/Bakdeficient cells, suggesting that particular signaling molecule(s) may be necessary for autophagic death, which is only activated by some cytotoxic stimuli.

It is also noteworthy that inhibition of caspases in WT MEFs treated with cytotoxic drugs inhibits apoptotic death but does not induce autophagic death. ${ }^{8}$ On the other hand, overexpression of $\mathrm{Bcl}-2$ or $\mathrm{Bcl}-\mathrm{x}_{\mathrm{L}}$ in WT MEFs (which is equivalent to Bax/Bak-deficiency in MEFs in terms of inhibiting apoptosis) blocks apoptosis but promotes autophagic death. ${ }^{8}$ These results imply that induction of autophagic death is not merely a consequence of the inhibition of apoptosis, but rather is regulated by the Bcl-2 family of proteins. This observation taken together with the finding that inhibitors of mitochondrial functions, such as respiratory chain inhibitors, can block autophagic death (our unpublished 
Withdrawal

of serum, amino acids or IL3 etc.

(+) Bax/Bak

(-) Bax/Bak

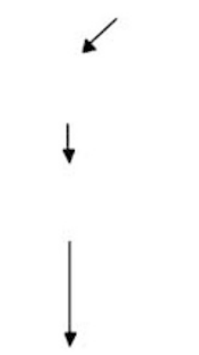

apoptosis

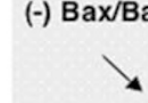

Atg6 (low)

Atg5 (low)

autophagy

survival
Etoposide, STS etc.

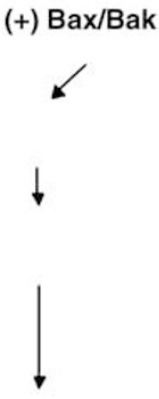

apoptosis

\section{(-) Bax/Bak}

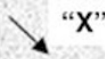

Atg6 (high) $\leftarrow \mathrm{Bcl}-2 / \mathrm{x}_{\mathrm{L}}$

Atg5 (high)

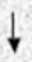

autophagy

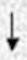

autophagic cell death

Figure 6 Autophagy and autophagic death. All stimuli (withdrawal of serum, amino acids, or lymphokines, and cytotoxic drugs such as etoposide and staurosporine) induce apoptosis of WT MEFs. When apoptosis is blocked by Bax/Bak-deficiency, only etoposide and staurosporine upregulate Atg5/Atg6 and activate autophagic death, whereas withdrawal of amino acids, serum, or lymphokines activate autophagy to prolong cell survival. Bcl-2/Bcl- $\mathrm{x}_{\mathrm{L}}$ is required for Bax/Bak-deficient MEFs to undergo autophagic death and might enhance the autophagic process via interaction with Atg6. STS: staurosporine

results) suggests that the mitochondria need to be functioning to allow the induction of autophagic death at least in MEFs. This might be contrary to the situation in Drosophila, where autophagic activity is seen in dying cells of the salivary gland and mid guts along with activation of caspases. ${ }^{15-17}$ Such findings imply that regulation of autophagic death varies considerably among different cells. In the fly, mitochondrial function might somehow be preserved even after caspase activation so that autophagic death occurs. Alternatively, autophagy may occur to more efficiently degrade the structural components of dying cells, and reduce the workload for phagocytes, and may not be a mechanism of cell death.

Studies performed with several culture systems have provided evidence that cells possess a novel death mechanism that depends on autophagy proteins. ${ }^{8,9}$ However, it can be argued that these are all nonphysiological models, so these findings do not necessarily indicate the physiological occurrence of this form of cell death. Of course, there are a number of in vivo situations where apoptosis is blocked at the mitochondrial level, such as upregulation of $\mathrm{Bcl}-2$ or $\mathrm{Bcl}-\mathrm{x}_{\mathrm{L}}$. So, our findings imply that these cells may have the potential to undergo autophagic death. In any case, although the results should be treated with caution, these culture systems provide an opportunity to investigate the autophagic death in detail, and such studies provide new evidence about the physiological and pathological occurrence of the autophagic death in vivo.

\section{Differentiating Autophagic Death from Autophagy}

The same set of the proteins (Atg5, Atg6, and Atg7) and the formation of autophagosomes are involved in both autophagic cell death and autophagy that promotes cell survival. ${ }^{8,9}$ Therefore, a question arises as to how these two opposite outcomes are regulated. Bax/Bak-deficient MEFs show activation of autophagy in response to both starvation and drug treatment, thus providing a useful tool to answer this question. One hint revealed by our recent study is a significant difference in the expression of Atg5 and Atg6 proteins during these two processes, since the levels of both proteins remain low during autophagy (survival), whereas these proteins are highly upregulated during autophagic death. ${ }^{8}$ Thus, it seems that the same set of proteins is involved in both autophagic death and autophagy (survival), but their regulation is substantially different during each process (Figure 6). Certainly, it needs to be experimentally proven whether this difference in the expression of Atg5 and Atg6 proteins leads to the selection of autophagic death or autophagy that promotes cell survival.

Depletion of amino acids or serum from WT MEFs induces apoptosis, but if apoptosis is blocked (e.g. by Bax/Bakdeficiency), activation of autophagy provides the nutrients necessary for cells to survive. Therefore, blocking autophagy causes cell death to be enhanced (see Figures 5 and 6). On the other hand, drugs such as etoposide or staurosporine upregulate the expression of some Atg proteins, leading to Atg protein-dependent cell death (Figures 5 and 6). It was recently shown that IL-3 deprivation induced autophagy in an IL-3dependent myeloid cell line, but death was not dependent on autophagy, because its inhibition actually enhanced cell death. ${ }^{14}$ Thus, IL-3 deprivation is similar to depletion of amino acids, in terms of creating a lack of something necessary for cell survival. Depletion of IL-3 is known to downregulate glucose uptake, eventually leading to an energy crisis ${ }^{14}$ that causes the activation of autophagy so that cells can obtain energy. Therefore, the inhibition of autophagy under conditions of IL-3 deprivation or amino-acid depletion enhances cell death due to exacerbation of the energy crisis or shortage of essential components. On the other hand, when Bax/Bakdeficient cells are exposed to etoposide or staurosporine, an 
energy crisis may not occur, judging from the observation that inhibition of autophagy improves cell viability. Thus, autophagy is activated in response to etoposide or staurosporine and leads to death by a totally different mechanism to that of autophagy related to cell survival. We think that autophagy may be activated in two different ways, which are (1) by depletion of factors that are necessary for cell survival and (2) by exposure to cytotoxic drugs or other stimuli that do not cause an energy crisis.

To explain this difference in the response, we hypothesize the existence of a specific signaling molecule ' $X$ ' that eventually causes the upregulation of Atg5 and Atg6 (Figure 6). ' $X$ ' is probably activated by several cytotoxic stimuli, such as etoposide and staurosporine, but not by depletion of amino acids, serum, or lymphokines such as IL-3, although all of these stimuli can trigger apoptosis in normal cells. Detection of ' $X$ ' will be crucial for a better understanding of the molecular basis of the signaling pathway involved in autophagic death, as well as for providing a specific marker that allows us to readily distinguish autophagic death from autophagy (survival). Autophagic death is clearly seen after exposure to cytotoxic drugs only when apoptosis is inhibited. However, there might be a signal that specifically activates the autophagic death program in vivo, probably through ' $\mathrm{X}$ ', without having an effect on the apoptotic machinery.

\section{Signaling Pathway: Possible Involvement of $\mathrm{Bcl}-2$ and JNK}

Information concerning the molecular basis of autophagic death is extremely limited. All we currently know is that this form of cell death is dependent on autophagy proteins that are involved in the formation of autophagosomes. ${ }^{8,9}$ Consistent with the process of autophagy, Atg6 acts upstream of Atg5 during autophagic death. ${ }^{8}$

The following questions remain to be answered: (1) How is the autophagic process activated by cytotoxic stimuli? (2) How are the processes of autophagy (related to cell survival) and autophagic death regulated? (3) Do autophagosomes sequester specific targets that lead to cell death? (4) Why does autophagy sometimes promote cell survival, but causes death under other circumstances?

One intriguing finding is that the autophagic death of Bax/ Bak-deficient MEFs requires the antiapoptotic proteins $\mathrm{Bcl}-\mathrm{x}_{\mathrm{L}}$ or $\mathrm{Bcl}-2^{8}$ (also refer to Figure 4). Certainly, it is necessary to determine whether this observation also applies to other cells. As Atg6 is required for the autophagic death of Bax/Bakdeficient cells ${ }^{8}$ and because $\mathrm{Bcl}-2 / \mathrm{Bcl}-\mathrm{x}_{\mathrm{L}}$ (but not Bax/Bak) binds to Atg6 (also called Beclin 1 that was initially identified as a $\mathrm{Bcl}-2$-binding protein), ${ }^{18} \mathrm{Bcl}-2 / \mathrm{Bcl}-\mathrm{x}_{\mathrm{L}}$ might influence the creation of autophagosomes at least partly via regulation of Atg6. This notion is supported by the finding that an cytotoxic drug-induced increase of Atg5-Atg12 in Bax/Bak-deficient MEFs (which is regulated by Atg6) is markedly reduced by silencing of $\mathrm{Bcl}-\mathrm{x}^{8}{ }^{8}$ This hypothesis needs to be tested by using mutants of $\mathrm{Bcl}-2 / \mathrm{Bcl}-\mathrm{x}_{\mathrm{L}}$ and Beclin 1 that cannot interact with each other.

Lenardo's group has shown that autophagic death of L929 cells is dependent on $\mathrm{JNK}^{9},{ }^{9}$ although they did not determine how JNK and the autophagy machinery were linked. We have confirmed a crucial role of JNK activation in the autophagic death of Bax/Bak-deficient MEFs (our unpublished results), while autophagy that promotes cell survival does not depend on JNK (our unpublished results). Therefore, activation of JNK might be a useful starting point for investigating the signaling pathway of autophagic death.

\section{Role of Autophagic Death in Physiological and Pathological Cell Death}

It has been suggested that autophagic death may play a role in both physiological and pathological cell death. This issue has been addressed by some recent reviews. ${ }^{19,20}$ However, an important point to remember is that the existing evidence about the role of autophagic death in physiological and pathological cell death processes is rather weak, because it is mainly based on morphologic studies. Therefore, these findings are not discussed here - readers who are interested should refer to the above-mentioned reviews. In this context, discussion of a few points might be useful in terms of setting the direction for future studies on autophagic death.

Autophagic death might be a phylogenetically ancient process, because 'morphologically defined' autophagic death is observed in lower eukaryotes such as nematodes, flies, and slime molds. ${ }^{21-24}$ Since genetic manipulation of all these organisms is possible, compelling evidence about the role of autophagic death in PCD should be obtained in near future. 'Morphologically defined' autophagic death has been described in the field of developmental biology. It seems that autophagic death is common during tissue remodeling processes, such as metamorphosis in insects and organ morphogenesis during development. ${ }^{7}$ Since autophagy can provide essential nutrients necessary for cells to survive, it is conceivable that autophagy may be used to provide nutrients to other cells in multicellular organisms. This could be an economical way for the remaining cells to reuse the components of organs or tissues that are removed during tissue remodeling and organ morphogenesis. What advantages might autophagic death have over apoptotic death as a form of PCD? It is conceivable that some PCD paradigms may destroy a massive number of cells, so that phagocytes might not be able to remove all of the apoptotic cells and debris. In contrast, the autophagic process could reduce the workload of phagocytes. At any rate, we still need to provide compelling evidence of the direct role of autophagic death in the physiological settings. Since mice are now available with deficiency of autophagy genes, such as Atg6-, Atg5- or Atg7deficient mice ${ }^{25-28}$ we could utilize such animals to verify that autophagic death plays a role in physiological PCD. Since yeast cells do not possess highly similar apoptotic machinery, but still die in the presence of cytotoxic drugs, it might be of interest to examine whether yeast cells undergo autophagic death.

It is very well established that the inhibition of apoptosis is a critical event in tumorigenesis. Elimination of cancer cells might not only occur via apoptosis but could also be mediated by other forms of cell death such as autophagic death. Some recent observations indicate that a decline of autophagic 
activity is related to tumorigenesis. ${ }^{25,26,29,30}$ Most importantly, it has been reported that heterozygosity of the atg 6 gene significantly increases tumor development in mice. ${ }^{25,26}$ Since it has been shown that autophagy plays a role in antigen presentation, this process might be important for the presentation of tumor antigens ${ }^{31}$ and inhibition of autophagy may allow tumor cells to escape immune surveillance. Alternatively, it has been shown that inhibition of autophagy increases the mutation rate in yeasts, although the underlying mechanism is unknown. If this also occurred in mammalian cells, an increase of the mutation rate by inhibition of autophagy in atg6 heterozygous mice would enhance tumor formation. It has also been suggested that inhibition of autophagy may increase cell proliferation. ${ }^{26}$ However, there is also the possibility that autophagic death eliminates tumor cells, so its inhibition would facilitate tumorigenicity as does the inhibition of apoptosis.

To better understand the pathology and the potential therapeutic strategies for human diseases that are characterized by enhanced cell death, it is crucial to elucidate the death mechanism(s) directly involved in each of these diseases. Cell death associated with human diseases could occur via apoptosis, necrosis, autophagic death, or other mechanisms that are currently unknown. Treatment should depend on the actual mechanism of cell death that plays a role in disease onset. There have been some reports suggesting the involvement of autophagic death in various diseases, since cells with autophagic features are found in the lesions of some neurodegenerative diseases, such as Parkinson disease and Alzheimer disease, ${ }^{7,32,33}$ as well as in some forms of myopathy. ${ }^{33,34,35}$

\section{Conclusion and Future Prospects}

PCD does not seem to be confined to apoptosis. Recent studies, ${ }^{8,9}$ although based on nonphysiological models, indicate that cells possess a mechanism of PCD that is associated with the formation of autophagosomes and depends on autophagy proteins - this is the true meaning of autophagic PCD. Although it will not be easy, it is now important to determine whether and to what extent autophagic death is involved when PCD occurs in vivo. It is also crucial to determine how apoptosis and autophagic death are regulated. Since there is a distinct possibility that autophagic death is involved in various human diseases, it would be invaluable to understand the molecular basis of autophagic death, so that new therapeutic strategies can be developed. Investigation of autophagic death is still in its infancy, but recently described culture systems, ${ }^{8,9}$ that allow autophagic death to be analyzed in detail, should be useful tools for studying this form of cell death under physiological and pathological conditions.

\section{Acknowledgements}

The work performed in my laboratory was supported in part by a grant for Center of Excellence Research, a grant for the 21st century COE Program, a grant for Scientific Research from the Ministry of Education, Science, Sports, and Culture of Japan, and by a grant for Research on
Dementia and Fracture from the Ministry of Health, Labor, and Welfare of Japan. We thank Ms Nao Tsujimoto for editorial assistance.

\section{References}

1. Schweichel JU and Merker HJ (1973) The morphology of various types of cell death in prenatal tissues. Teratology 7: 253-266

2. Ohsumi $Y$ (2001) Molecular dissection of autophagy: two ubiquitin-like systems. Nat. Rev. Mol. Cell. Biol. 2: 211-216

3. Huang WP and Klionsky DJ (2002) Autophagy in yeast: a review of the molecular machinery. Cell Struct. Funct. 27: 409-420

4. Mizushima N, Ohsumi Y and Yoshimori T (2002) Autophagosome formation in mammalian cells. Cell Struct. Funct. 27: 421-429

5. Gutierrez MG, Master SS, Singh SB, Taylor GA, Colombo Ml and Deretic V (2004) Autophagy is a defense mechanism inhibiting BCG and Mycobacterium tuberculosis survival in infected macrophages. Cell 119: 753-766

6. Ogawa M, Yoshimori T, Suzuki T, Sagara H, Mizushima N and Sasakawa C (2005) Escape of intracellular Shigella from autophagy. Science 307: 727-731

7. Bursch W (2001) The autophagosomal-lysosomal compartment in programmed cell death. Cell Death Differ. 8: 569-581

8. Shimizu S, Kanaseki T, Mizushima N, Mizuta K, Arakawa-Kobayashi S, Thompson CB and Tsujimoto Y (2004) A role of Bcl-2 family of proteins in nonapoptotic programmed cell death dependent on autophagy genes. Nat. Cell Biol. 6: 1221-1228

9. Yu L, Alva A, Su H, Dutt P, Freundt E, Welsh S, Baehrecke EH and Lenardo MJ (2004) Regulation of an ATG7-Beclin 1 program of autophagic cell death by caspase-8. Science 304: 1500-1502

10. Lindsten T, Ross AJ, King A, Zong WX, Rathmell JC, Shiels HA, Ulrich E, Waymire KG, Mahar P, Frauwirth K, Chen Y, Wei M, Eng VM, Adelman DM, Simon MC, Ma A, Golden JA, Evan G, Korsmeyer SJ, MacGregor GR and Thompson CB (2000) The combined functions of proapoptotic Bcl-2 family members bak and bax are essential for normal development of multiple tissues. Mol. Cell 6: 1389-1399

11. Wei MC, Zong WX, Cheng EH, Lindsten T, Panoutsakopoulou V, Ross AJ, Roth KA, MacGregor GR, Thompson CB and Korsmeyer SJ (2001) Proapoptotic BAX and BAK: a requisite gateway to mitochondrial dysfunction and death. Science 292: 727-730

12. Yue Z, Horton A, Bravin M, DeJager PL, Selimi F and Heintz N (2002) A novel protein complex linking the delta 2 glutamate receptor and autophagy: implications for neurodegeneration in lurcher mice. Neuron 35: 921-933

13. Pyo JO, Jang MH, Kwon YK, Lee HJ, Jun JI, Woo HN, Cho DH, Choi B, Lee H, Kim JH, Mizushima N, Oshumi Y and Jung YK (2005) Essential roles of Atg5 and FADD in autophagic cell death: dissection of autophagic cell death into vacuole formation and cell death. J. Biol. Chem. 280: 20722-20729

14. Lum JJ, Bauer DE, Kong M, Harris MH, Li C, Lindsten T and Thompson CB (2005) Growth factor regulation of autophagy and cell survival in the absence of apoptosis. Cell 120: 237-248

15. Lee CY and Baehrecke EH (2001) Steroid regulation of autophagic programmed cell death during development. Development 128: 1443-1455

16. Martin DN and Baehrecke EH (2004) Caspases function in autophagic programmed cell death in Drosophila. Development 131: 275-284

17. Lee CY, Cooksey BA and Baehrecke EH (2002) Steroid regulation of midgut cell death during Drosophila development. Dev. Biol. 250: 101-111

18. Liang XH, Kleeman LK, Jiang HH, Gordon G, Goldman JE, Berry G, Herman B and Levine B (1998) Protection against fatal Sindbis virus encephalitis by Beclin, a novel Bcl-2-interacting protein. J. Virol. 72: 8586-8596

19. Gozuacik D and Kimchi A (2004) Autophagy as a cell death and tumor suppressor mechanism. Oncogene 23: 2891-2906

20. Levine B and Klionsky DJ (2004) Development by self-digestion: molecular mechanisms and biological functions of autophagy. Dev. Cell 6: 463-477

21. Cornillon S, Foa C, Davoust J, Buonavista N, Gross JD and Golstein P (1994) Programmed cell death in Dictyostelium. J. Cell Sci. 107 (Part 10): 2691-2704

22. Olie RA, Durrieu F, Cornillon S, Loughran G, Gross J, Earnshaw WC and Golstein $P$ (1998) Apparent caspase independence of programmed cell death in Dictyostelium. Curr. Biol. 8: 955-958

23. Hall DH, Gu G, Garcia-Anoveros J, Gong L, Chalfie M and Driscoll M (1997) Neuropathology of degenerative cell death in Caenorhabditis elegans. J. Neurosci. 17: 1033-1045 
24. Baehrecke EH (2003) Autophagic programmed cell death in Drosophila. Cell Death Differ. 10: 940-945

25. Yue Z, Jin S, Yang C, Levine AJ and Heintz N (2003) Beclin 1, an autophagy gene essential for early embryonic development, is a haploinsufficient tumor suppressor. Proc. Natl. Acad. Sci. USA 100: 1507715082

26. Qu X, Yu J, Bhagat G, Furuya N, Hibshoosh H, Troxel A, Rosen J, Eskelinen EL, Mizushima N, Ohsumi Y, Cattoretti G and Levine B (2003) Promotion of tumorigenesis by heterozygous disruption of the Beclin 1 autophagy gene. J. Clin. Invest. 112: 1809-18020

27. Kuma A, Hatano M, Matsui M, Yamamoto A, Nakaya H, Yoshimori T, Ohsumi Y, Tokuhisa T and Mizushima N (2004) The role of autophagy during the early neonatal starvation period. Nature 432: 1032-1036

28. Komatsu M, Waguri S, Ueno T, Iwata J, Murata S, Tanida I, Ezaki J, Mizushima N, Ohsumi Y, Uchiyama Y, Kominami E, Tanaka K and Chiba T (2005) Impairment of starvation-induced and constitutive autophagy in Atg7-deficient mice. J. Cell Biol. 169: 425-434

29. Kisen GO, Tessitore L, Costelli P, Gordon PB, Schwarze PE, Baccino FM and Seglen PO (1993) Reduced autophagic activity in primary rat hepatocellular carcinoma and ascites hepatoma cells. Carcinogenesis 14: 2501-2505

30. Liang XH, Jackson S, Seaman M, Brown K, Kempkes B, Hibshoosh H and Levine $B$ (1999) Induction of autophagy and inhibition of tumorigenesis by Beclin 1. Nature 402: 672-676

31. Paludan C, Schmid D, Landthaler M, Vockerodt M, Kube D, Tuschl T and Munz C (2005) Endogenous MHC class II processing of a viral nuclear antigen after autophagy. Science 307: 593-596

32. Anglade P, Vyas S, Javoy-Agid F, Herrero MT, Michel PP, Marquez J, MouattPrigent A, Ruberg M, Hirsch EC and Agid Y (1997) Apoptosis and autophagy in nigral neurons of patients with Parkinson's disease. Histol. Histopathol. 12: 25-31

33. Bahr BA and Bendiske $J(2002)$ The neuropathogenic contributions of lysosomal dysfunction. J. Neurochem. 83: 481-489

34. Kaneda D, Sugie K, Yamamoto A, Matsumoto H, Kato T, Nonaka I and Nishino I (2003) A novel form of autophagic vacuolar myopathy with late-onset and multiorgan involvement. Neurology 61: 128-131

35. Kalimo H, Savontaus ML, Lang H, Paljarvi L, Sonninen V, Dean PB, Katevuo K and Salminen A (1988) X-linked myopathy with excessive autophagy: a new hereditary muscle disease. Ann. Neurol. 23: 258-265 